\title{
A 71-yr-old male with increasing dyspnoea, cough and an intrathoracic mass
}

\author{
E. Seebus*, B. Vrugt", R.S. de Jong*, R. Aalbers ${ }^{\natural}$
}

\section{Case history}

A 71-year-old White male was referred to the Martini Hospital for evaluation of progressive shortness of breath, tiredness, a nonproductive cough and night sweats. Until the beginning of his complaints a few weeks before, he felt healthy and skated $40 \mathrm{~km}$ per week. His appetite was good, he had not lost weight, had no skin or joint disorders and no thoracic pain.

His medical history revealed a partial gastrectomy and duodenectomy for persistent ulcers and bronchitis in his childhood. Exposure to asbestos was uncertain. There were no family related diseases. He did not use any medication, but had smoked over 50 pack-yrs and drank alcohol occasionally. He had been a cook in the navy and a swimming instructor.

Routine physical examination showed a vital, healthylooking man with finger clubbing, decreased breathing sounds and dullness to percussion at the dorsal inferior part of the right hemithorax. There were no palpable lymph nodes, no signs of osteoarthropathy and the liver was not enlarged.

Routine laboratory findings and arterial blood-gas analysis showed no abnormalities, except for the blood sedimentation rate of $71 \mathrm{~mm} \cdot \mathrm{h}^{-1}$ (normal $<10 \mathrm{~mm} \cdot \mathrm{h}^{-1}$ ), C-reactive protein of $32 \mathrm{mg} \cdot \mathrm{mL}^{-1}$ (normal $<8 \mathrm{mg} \cdot \mathrm{mL}^{-1}$ ), alkaline phosphatase of $260 \mathrm{U} \cdot \mathrm{L}^{-1}$ (normal $<130 \mathrm{U} \cdot \mathrm{L}^{-1}$ ) and $\gamma$-glutamyl transpeptidase of $85 \mathrm{U} \cdot \mathrm{L}^{-1}$ (normal $<50 \mathrm{U} \cdot \mathrm{L}^{-1}$ ). The electrocardiogram was normal.

A chest radiograph (fig. 1) was taken, followed by a bronchoscopy, which showed no abnormalities. Further investigation included ultrasonography, computed tomography (CT), magnetic resonance imaging (MRI) of the thorax (fig. 2) and angiography.

Spirography demonstrated a total lung volume capacity of $4.85 \mathrm{~L}$ ( $80 \%$ predicted), residual volume of $1.76 \mathrm{~L}$ (70\% pred), vital capacity of $3.02 \mathrm{~L}(91 \%)$ and diminished forced expiratory volume in one second of $2.09 \mathrm{~L}(82 \%)$. Histology of the tumour (fig. 3) was obtained after surgery.

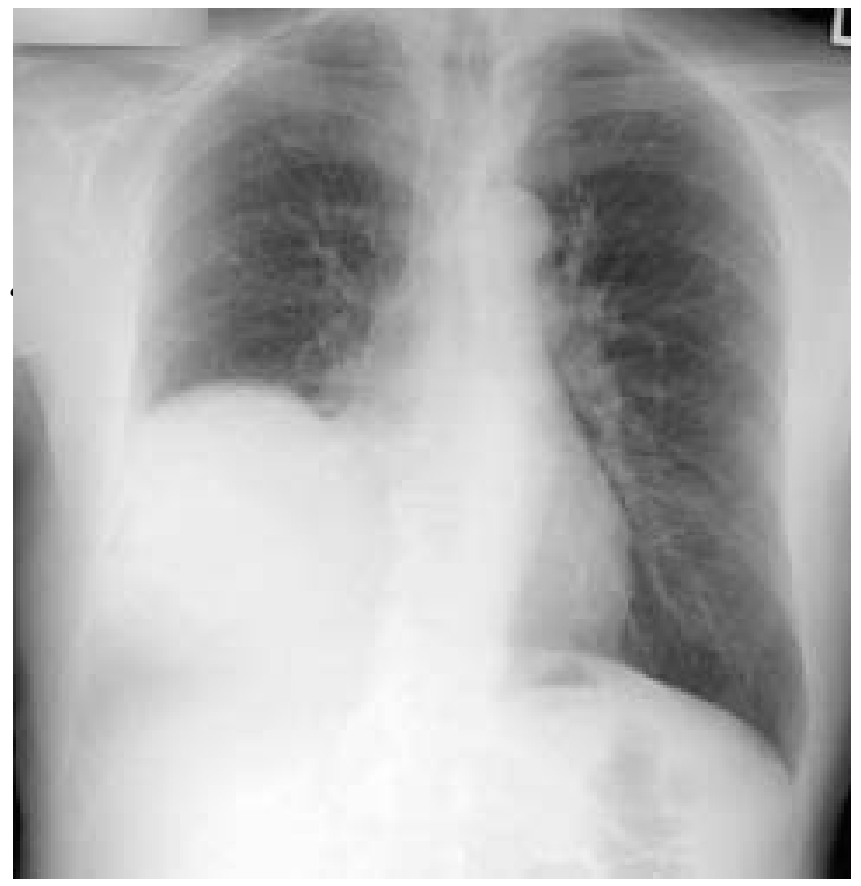

Fig. 1.-Chest radiograph: frontal projection.

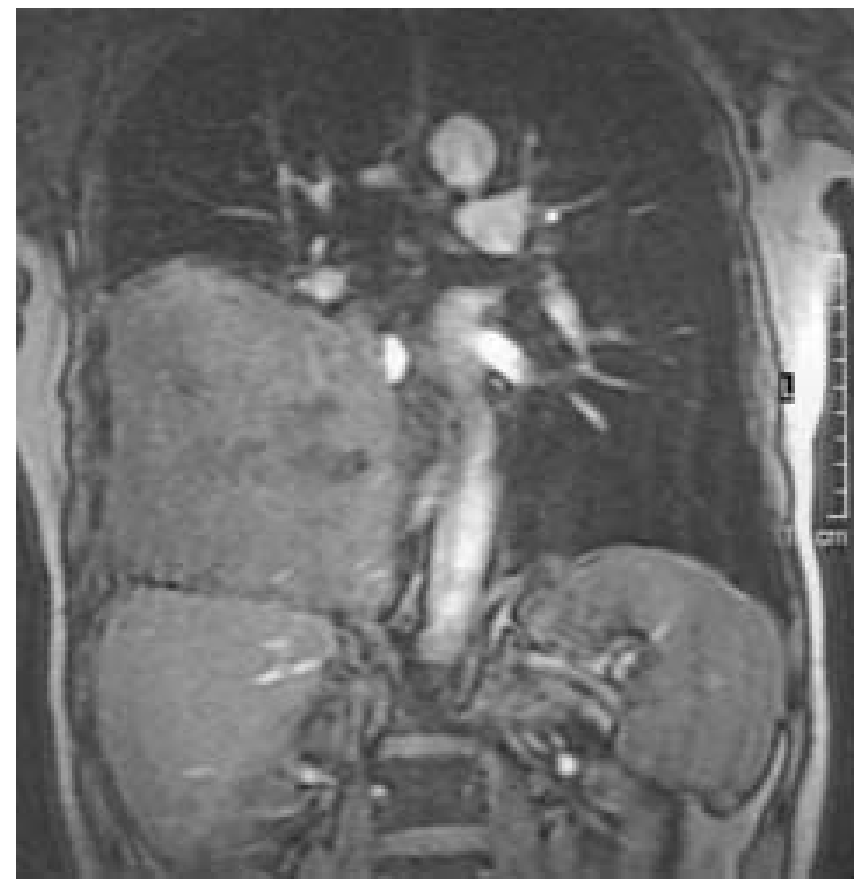

Fig. 2. - Magnetic resonance imaging of the thorax.

\footnotetext{
*Dept of Internal Medicine, ${ }^{\#}$ Dept of Pathology, and "Dept of Pulmonology, Martini Hospital, Groningen, the Netherlands. Correspondence: R. Aalbers, Dept of Pulmonology, Martini Hospital, Postbox 30033, 9700 RM Groningen, the Netherlands. Fax: 31505245937. E-mail: r.aalbers@mzh.nl
} 

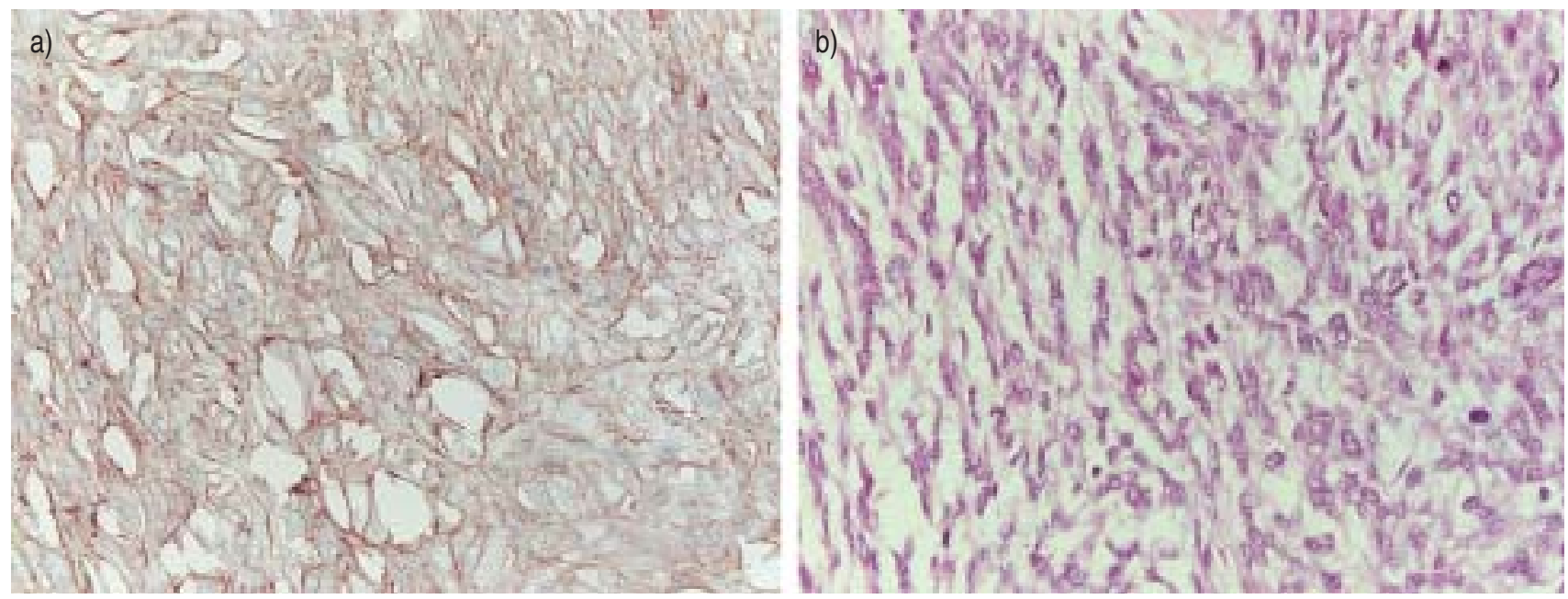

Fig. 3. - Histology of the tumour. a) Haematoxylin and eosin staining and b) immunohistochemical staining for CD34.

BEFORE TURNING THE PAGE, INTERPRET THE CHEST RADIOGRAPH, THE MAGNETIC RESONANCE IMAGE AND THE HISTOLOGICAL STUDIES OF THE TUMOUR, AND SUGGEST A DIAGNOSIS. 


\section{Interpretation}

The laboratory findings showed slightly elevated inflammatory parameters and biliary peptides.

The chest radiograph, ultrasonography and CT revealed a large part of the right hemithorax was filled with a nonhomogeneous solid tumour, apparently consisting of two compartments, closely related to the right hilus, but free of large vessels. The relationship to the diaphragm and the involvement of other structures could not be assessed. No enlarged mediastinal lymph nodes were seen.

These findings were confirmed by MRI (fig. 2). Furthermore, MRI did not demonstrate tumour growth into the oesophagus or heart, although involvement of the diaphragm could not be excluded. Angiography showed moderate vascularisation, possibly originating from branches of the intercostal arteries. Fortunately, no blood supply from the abdomen was found, as this would increase the operation risk.

Concluding, a large, solid, nonhomogeneous, highly vascularised process in the lower lobe of the right lung was discovered. The process was expected to be benign, most likely being a pleurafibroma or a response to inflammation, because of the tumour size, and age, vitality and complaints of the patient. Of course, malignancy could not be excluded at this stage, so peripheral bronchial carcinoma, mesothelioma, solitary pleural metastasis, lymphoma, pleural fibrosarcoma and neurogenic tumour were also taken into consideration. Owing to the possibility of malignancy, percutaneous biopsy under CT guidance for histological diagnosis was not performed, thus avoiding contamination and consequently not decreasing curative options.

Spirography showed that a pneumectomy was feasible. Excision of the tumour was performed by thoracotomy and lobectomy of the right lower lobe.

Macroscopic examination showed a broadly based, lobulated tumour, $20 \mathrm{~cm}$ in diameter and $1,987 \mathrm{~g}$ in weight, originating from the visceral pleura. Cut section typically revealed a firm, glistening, grey-white surface with a whorled pattern. At microscopy (fig. 3), a highly vascularised mesenchymal tumour with variable cell densities was seen. The cellular areas consisted of densely packed fusiform nuclei and finely fibrillar collagen, alternated with sclerotic areas of coarsely fibrous collagen and infrequent nuclei. Although the tumour elevated and distorted the overlying pleura, the surface mesothelial cells remained intact, being continuous as a single layer of hypertrophical cells (capsule). A relatively high mitosis index of 17-27 per $2 \mathrm{~mm}^{2}$ was found in the more cellular parts of the lesion, and in some sections necrosis was prominent. Immunohistochemical examination showed a strong and diffuse expression of the tumour cells for CD34, bcl-2, but all keratins and CD31 were negative. The histomorphological and immunohistochemical findings were compatible with the clinical diagnosis of a solitary fibrous tumour. Owing to the high cell turnover and the presence of necrosis the lesion was considered to be malignant.

\section{Diagnosis: Malignant solitary fibrous tumour of the pleura}

\section{Clinical course}

The control chest radiograph after lobectomy showed good expansion of the remaining right lung, without abnormalities on the left side. Shortness of breath and finger clubbing disappeared. The patient is still alive $2.5 \mathrm{yrs}$ after diagnosis, although recent evaluation showed a tumour in the inferior part of the right hemithorax and at least four lesions in the left lung, suspect for return of solitary fibrous tumours of the pleura (SFTPs) with metastases.

\section{Discussion}

SFTPs are rare. Clinical presentation varies, depending on size and intrathoracic localisation. Most patients have little or no symptoms at the time of presentation. Often the tumour is noticed on a routine chest radiograph. SFTPs may enlarge rapidly, causing dyspnoea, cough, chest pain, and may induce paraneoplastic syndromes, such as hypoglycaemia (related to pathological secretion of a precursor of insulin-like growth factor II by the tumour), digital clubbing and osteochondropathy [1]. Although aggressive forms have been described with local infiltration, distant metastases and local recurrence, the majority of solitary fibrous tumours are histologically benign. SFTPs are not associated with asbestos [2].

SFTPs usually present as intrathoracic, usually homogeneous, sharply delineated round or lobulated masses, sometimes associated with ipsilateral pleural effusion or pulmonary atelectasis [3]. Tumours may be nonhomogeneous, due to necrosis, haemorrhage within the tumour, or myxoid degeneration. MRI can be used to assess tumour extent and vascular or chest wall invasion. Angiography is not recommended in the literature as a radiological tool in the work-up of SFTPs. However, more detailed information about vascularisation of these tumours before surgery may prevent heavy bleeding during excision.

SFTPs originate from submesothelial connective tissue. These fibrous tumours have typical cytological and immunocytochemical features (see interpretations), which allow diagnosis on tissue biopsy preparations [4]. A cytological diagnosis of SFTPs is possible if specimens contain vimentinpositive and CD34-positive cells with dendritic/stellate or spindle/bipolar morphology [5]. However, cell morphology of solitary fibrous tumours varies widely and often too little cells are retrieved, moreover, tumour spill is being risked at biopsy. Spindle cells characteristically have sporadic or absent mitoses, however, in larger tumours a high mitosis index is observed [2].

Treatment is always surgical. Conclusive diagnosis is retrieved by histology, generally after excision of the tumour. Video-assisted thoracic surgery, with intra-operative assessment of the surgical margins, is the treatment of choice [2], although this can be questioned for such large tumours. Positive surgical margins are associated with recurrence. Complete resection is the best prognostic factor [6], and paraneoplastic syndromes recede after surgery [3]. Most patients with a solitary fibrous tumour fare well after surgical treatment, although clinical and radiological follow-up are indicated. Closer observation is justified for tumours measuring $>10 \mathrm{~cm}$ in diameter, because this predicts shorter diseasefree survival and a high tendency of local recurrence and/or metastasis [7].

The patient described in this case report suffered from a malignant solitary fibrous tumour of the pleura. This is an extremely rare fibrous tumour. It may be locally aggressive and is capable of invading adjacent structures, decreasing resectability. In addition, the malignant form reappears more often than the benign type. The best prognostic factor for malignant solitary fibrous tumours of the pleura is complete resection. Radiotherapy for this malignant tumour is not recommended. 


\section{References}

1. Meyer M, Krause U. Solitary fibrous tumors of the pleura. Chirurg 1999; 70: 949-952.

2. Briselli M, Mark EJ, Dickersin GR. Solitary fibrous tumors of the pleura: eight new cases and review of 360 cases in the literature. Cancer 1981; 47: 2678-2689.

3. Rena O, Filosso PL, Papalia E, et al. Solitary fibrous tumour of the pleura: surgical treatment. Eur J Cardiothorac Surg 2001; 19: 185-189.

4. Hummel P, Cangiarella JF, Cohen JM, Yang G, Waisman J, Chieng DC. Transthoracic fine-needle aspiration biopsy of pulmonary spindle cell and mesenchymal lesions: a study of 61 cases. Cancer 2001; 25; 93: 187-198.

5. Okada S, Ebihara Y, Kudo M, et al. Scratch cytologic findings on surgically resected solitary fibrous tumors of the pleura. Acta Cytol 2001; 45: 372-380.

6. Magdeleinat $\mathrm{P}$, Alifano M, Petino A, et al. Solitary fibrous tumors of the pleura: clinical characteristics, surgical treatment and outcome. Eur J Cardiothorac Surg 2002; 21: 1087-1093.

7. Gold JS, Antonescu CR, Hajdu C, et al. Clinicopathologic correlates of solitary fibrous tumors. Cancer 2002; 94: 10571068 . 